\title{
Dietary 7-Ketocholesterol Exacerbates Myocardial Ischemia-Reperfusion Injury in Mice through Monocyte/Macrophage-Mediated Inflammation
}

\section{Tomoki Uchikawa}

Kyushu University

Tetsuya Matoba ( $\nabla$ matoba@cardiol.med.kyushu-u.ac.jp )

Kyushu University

Takuro Kawahara

Kyushu University

Isashi Baba

Kyushu University

Shunsuke Katsuki

Kyushu University

Jun-ichiro Koga

Kyushu University

Yu Hashimoto

Kyushu University

Ryo Yamasaki

Kyushu University

Ikuyo Ichi

Ochanomizu University

Hidetaka Akita

Chiba University

Hiroyuki Tsutsui

Kyushu University

Research Article

Keywords:

Posted Date: March 7th, 2022

DOI: https://doi.org/10.21203/rs.3.rs-1360401/v1 
License: (c) (i) This work is licensed under a Creative Commons Attribution 4.0 International License. Read Full License 


\section{Abstract}

Background: Emerging evidence suggests that 7-ketocholesterol (7-KC), one of the most abundant dietary oxysterols, causes inflammation and cardiovascular diseases. Here we show the deteriorating effects of dietary 7-KC on myocardial ischemia-reperfusion (IR) injury and detailed the molecular mechanisms.

Methods and Results: A high-fat high-cholesterol diet containing 7-KC (7KWD) for 3 weeks increased the plasma 7-KC level compared with high-fat high-cholesterol diet in mice. In wild-type mice but not in $\mathrm{CCR}^{-/-}$mice, dietary 7-KC increased the myocardial infarct size after IR. Flow cytometry revealed that the

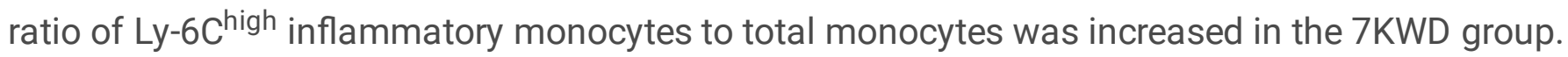
Unbiased RNA sequencing using murine primary macrophages revealed that 7-KC regulated the expression of transcripts related to inflammation and cholesterol biosynthesis. We further validated that in vitro, 7-KC induced endoplasmic reticulum stress, mitochondrial reactive oxygen species production, and nuclear factor-kappa B activation, which are associated with increased mRNA levels of proinflammatory cytokines. Administration of $\mathrm{N}$-acetyl-L-cysteine or siRNA-mediated knockdown of PKRlike endoplasmic reticulum kinase or endoplasmic reticulum oxidase 1a suppressed the levels of 7-KCinduced inflammation.

Conclusion: Dietary 7-KC exacerbates myocardial IR injury through monocyte/macrophage-mediated inflammation. Endoplasmic reticulum stress and oxidative stress are involved in the 7-KC-induced proinflammatory response in macrophages.

\section{Introduction}

Acute myocardial infarction (AMI) is the most devastating complication of coronary atherosclerosis. ${ }^{1}$ Post-infarct left ventricular remodeling causes heart failure and reduces quality of life. ${ }^{2-4}$ While primary percutaneous coronary intervention $(\mathrm{PCl})$ reduces mortality after ST-segment elevation myocardial infarction (STEMI), ${ }^{3,4}$ the recent shortening of door-to-balloon time has not reduced in-hospital mortality. ${ }^{5}$ Ischemia-reperfusion (IR) injury induces cardiomyocyte death and limits the beneficial effects of $\mathrm{PCl}{ }^{6,7}$ Hence, improving the understanding of the detailed molecular mechanisms of myocardial IR injury and developing novel therapeutics targeting IR injury are promising therapy-related strategies to improve the prognosis of STEMI patients.

Myocardial IR injury is mainly due to mitochondrial dysfunction and myocardial inflammation mediated by monocytes/macrophages promoting cell death. ${ }^{6,8,9}$ A proinflammatory monocyte subset, Ly- $6 \mathrm{C}^{\text {high }}$ monocytes, exacerbates inflammation and subsequent myocardial IR injury in mice. ${ }^{8-10}$ We previously reported that the deficiency of CCR2, a receptor for MCP-1 that mediates monocyte/macrophage chemotaxis, as well as nanoparticle-mediated delivery of an angiotensin II type 1 receptor blocker, ${ }^{11}$ a peroxisome proliferator-activated receptor $y$ agonist, ${ }^{12}$ or a toll-like receptor 4 (TLR4) blocker ${ }^{13}$ to the monocytes in the blood and IR heart ameliorated myocardial IR injury by inhibiting the recruitment of Ly$6 \mathrm{C}^{\text {high }}$ monocytes to the IR heart. 
Current lipid-lowering therapies involving statins, ezetimibe, and proprotein convertase subtilisin/kexin type-9 (PCSK-9) inhibitors have been established as standard therapies for coronary artery disease (CAD) ${ }^{14,15}$ There, however, remains a substantial residual risk of CAD even with the administration of these intensive lipid-lowering therapies. ${ }^{16}$ Dietary oxysterols produced by nonenzymatically oxidized sterol rings during storage or cooking are an important residual risk factor for CAD. ${ }^{17}$ Several oxysterols are reported to induce oxidative stress, inflammation, and cell death. ${ }^{18-21}$ We also demonstrated that oxysterols promoted atherosclerosis in mice ${ }^{22}$ and coronary endothelial dysfunction in patients with CAD. ${ }^{23}$ The absolute amount of oxysterols in the blood, including 7-ketocholesterol (7-KC), is very low, at approximately $0.1 \%$ of the amount of cholesterol; ${ }^{23-25}$ however, $7-\mathrm{KC}$ is one of the most common dietary oxysterols and the main component of oxidized cholesterol in oxidized low-density lipoprotein ${ }^{26,27}$ and correlates with cardiovascular outcomes and total mortality in patients with CAD. ${ }^{28} 7-K C$ is produced by nonenzymatic oxidization at the $\mathrm{C} 7$ position of cholesterol, which changes the hydrogen to a ketone. ${ }^{29}$ We previously demonstrated that 7-KC possesses proinflammatory properties in vascular cells, such as promoting endothelial cell proliferation and tissue factor expression in smooth muscle cells. ${ }^{25}$ However, the molecular mechanisms by which 7-KC induces inflammation in macrophages are not fully understood, and it has not been clarified whether dietary 7-KC affects myocardial IR injury.

Therefore, we hypothesized that 7-KC can exacerbate myocardial IR injury by promoting monocyte/macrophage-mediated inflammation. The present study used a mouse model of myocardial IR injury to examine the effects of 7-KC on myocardial infarct size and the underlying mechanisms by which 7-KC exacerbates inflammation in the IR myocardium.

\section{Results}

\section{Dietary 7-KC exacerbated myocardial IR injury through monocyte/macrophage-mediated inflammation}

We first quantified plasma lipid and oxysterol levels after 3 weeks of feeding a normal laboratory diet, control western diet (WD), or WD containing 7-KC (7KWD). There were no significant differences in plasma cholesterol and triglyceride levels between the WD group and 7KWD group. Dietary 7-KC, however, markedly increased oxysterol levels, especially the levels of 7-KC and 7 $\beta$-hydroxycholesterol, a metabolite

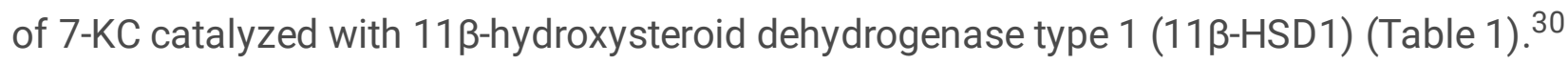

\section{Table 1. Plasma lipid parameters}




\begin{tabular}{|llll|}
\hline Lipid profiles & & & \\
& ND & WD & $7 K W D$ \\
& $(\mathrm{~N}=6)$ & $(\mathrm{N}=6)$ & $(\mathrm{N}=6)$ \\
\hline Total Cholesterol $(\mathrm{mg} / \mathrm{dL})$ & $93 \pm 5$ & $160 \pm 4^{\star \star \star}$ & $150 \pm 8^{\star \star \star}$ \\
\hline LDL Cholesterol $(\mathrm{mg} / \mathrm{dL})$ & $20 \pm 1$ & $37 \pm 2^{\star \star \star}$ & $36 \pm 1^{\star \star \star}$ \\
\hline HDL Cholesterol $(\mathrm{mg} / \mathrm{dL})$ & $54 \pm 2$ & $41 \pm 3^{\star \star}$ & $44 \pm 2^{*}$ \\
\hline Triglyceride $(\mathrm{mg} / \mathrm{dL})$ & $84 \pm 8$ & $23 \pm 1^{\star \star \star}$ & $27 \pm 4^{\star \star \star}$ \\
\hline
\end{tabular}

Oxysterols

\begin{tabular}{|c|c|c|c|}
\hline & $\begin{array}{l}\mathrm{ND} \\
(\mathrm{N}=8)\end{array}$ & $\begin{array}{l}\text { WD } \\
(\mathrm{N}=8)\end{array}$ & $\begin{array}{l}7 K W D \\
(N=8)\end{array}$ \\
\hline 7a-hydroxycholesterol & $8.9 \pm 3.7$ & $41.1 \pm 15.1$ & $17.3 \pm 6.4$ \\
\hline $7 \beta$-hydroxycholesterol & $54.0 \pm 20.1$ & $149.8 \pm 29.9$ & $1168.0 \pm 241.6^{\star \star \star+\dagger}$ \\
\hline 7-ketocholesterol & $64.5 \pm 12.5$ & $144.7 \pm 31.7$ & $399.4 \pm 61.9^{\star \star \star+\dagger}$ \\
\hline $5 \beta, 6 \beta$-epoxycholesterol & $14.3 \pm 8.5$ & $43.5 \pm 11.6$ & $31.2 \pm 6.4$ \\
\hline $4 \beta$-hydroxycholesterol & $79.0 \pm 9.6$ & $385.6 \pm 69.2^{* *}$ & $457.7 \pm 77.4^{\star \star \star}$ \\
\hline cholestan-3 $3,5 a, 6 \beta$-triol & $0.1 \pm 0.0$ & $0.3 \pm 0.1$ & $0.3 \pm 0.2$ \\
\hline 24-hydroxycholesterol & $3.9 \pm 1.3$ & $11.6 \pm 3.3$ & $25.6 \pm 4.4^{\star \star \star \dagger}$ \\
\hline 25-hydroxycholesterol & $18.3 \pm 5.2$ & $13.6 \pm 3.0$ & $17.5 \pm 2.9$ \\
\hline 27-hydroxycholesterol & $22.3 \pm 6.2$ & $53.1 \pm 11.0^{*}$ & $56.3 \pm 6.1^{*}$ \\
\hline Total oxysterols & $480 \pm 61$ & $1067 \pm 113$ & $2391 \pm 299^{\star \star \star+\dagger}$ \\
\hline
\end{tabular}

Cholesterol and triglyceride levels are shown in $\mathrm{mg} / \mathrm{dL}$. The level of each oxysterol component is shown in $\mathrm{ng} / \mathrm{mL}$. Data are expressed as the mean $\pm S E M$ and were compared using one-way ANOVA with the post hoc Tukey HSD test. ${ }^{*} p<0.05,{ }^{* *} p<0.01$, and ${ }^{* \star *} p<0.001$ versus the ND group. ${ }^{\dagger} p<0.05$, and ${ }^{+\dagger} p<0.001$ versus the WD group. ND, normal laboratory diet; WD, Western diet; 7KWD, WD containing 7-KC.

We next examined the effects of dietary 7-KC on myocardial IR injury. As shown in Figure S1 in the Data Supplement, there were no significant differences in the AAR/LV among the three groups. Importantly, dietary 7-KC increased the infarct size after myocardial IR with no significant effects on blood pressure or heart rate (Fig. 1A, Figure S2 in the Data Supplement). Immunostaining for Mac-3 revealed that the 7KWD 
increased the accumulation of Mac-3-positive cells compared to the WD after myocardial IR (Fig. 1B).

Flow cytometry revealed that the 7KWD increased the ratio of Ly- $6 C^{\text {high }}$ inflammatory monocytes to total monocytes in the heart after myocardial IR (Fig. 1C, Figure S3 in the Data Supplement). Previous studies have shown that deletion of CCR2 decreases infarct size in a murine myocardial IR injury model, suggesting that monocyte/macrophage-mediated inflammation may play a key role in the mechanisms by which 7-KC exacerbates myocardial IR injury. ${ }^{11}$ We therefore employed CCR2-deficient mice to clarify the relative importance of monocyte/macrophage-mediated inflammation in the underlying mechanisms. As shown in Fig. 1D, CCR2 deficiency abrogated the effect of 7-KC on the infarct size after myocardial IR. This result confirmed that dietary 7-KC exacerbated myocardial IR injury primarily through CCR2-positive monocyte/macrophage-mediated inflammation, not through direct effects on cardiomyocytes, after myocardial IR.

\section{7-KC facilitated macrophage differentiation into a proinflammatory phenotype and accelerated the macrophage migratory capacity by increasing CCR2 expression}

To elucidate the detailed mechanisms by which 7-KC promotes macrophage-mediated inflammation in the IR heart, we examined the effects of 7-KC on monocyte differentiation. Bone marrow cells isolated from WT mice were cultured with $20 \mathrm{ng} / \mathrm{mL}$ macrophage colony-stimulating factor (M-CSF) for 5 days to induce differentiation into bone marrow-derived macrophages (BMDMs). Pretreatment with 7-KC enhanced the induction of proinflammatory factors, including monocyte chemotactic protein 1 (MCP-1) and iNOS, by $10 \mathrm{ng} / \mathrm{mL}$ LPS and $10 \mathrm{ng} / \mathrm{mL}$ IFN- $\gamma$ compared to control pretreatment with cholesterol (Fig. $2 \mathrm{~A})$, suggesting that 7-KC facilitated macrophage differentiation into a proinflammatory phenotype. Next, we used bone marrow cells isolated from CX3CR1-GFP/CCR2-RFP mice to clarify the effects of 7-KC on chemokine receptor expression. In CX3CR1-GFP/CCR2-RFP mice, RFP is coexpressed with CCR2, and GFP is coexpressed with CX3CR1. ${ }^{31}$ As shown in Fig. 2B, 7-KC increased CCR2 expression in BMDMs, as determined from the fluorescence intensity of RFP, whereas there were no significant effects on GFP intensity, representing CX3CR1 expression. Finally, we examined the migratory capacity of peritoneal macrophages isolated from WT mice fed the WD or 7KWD. 7-KC increased the number of migrated macrophages in the 7KWD group compared to that in the WD group (Fig. 2C). Collectively, these results demonstrated that 7-KC promoted macrophage accumulation in the IR heart by increasing the expression of both MCP-1 and CCR2 in macrophages.

\section{Unbiased transcriptomic analysis revealed 7KC-induced activation of several key proinflammatory signaling pathways}


Because in vivo study suggested that monocyte/macrophage-mediated inflammation is responsible for the 7-KC-induced exacerbation of myocardial IR injury, we performed in vitro assays using murine primary macrophages. We explored the effects of 7-KC in an unbiased manner using RNA sequencing (RNA-Seq). RNA-Seq analysis of murine peritoneal macrophages treated with 7-KC revealed differentially expressed transcripts between the 7-KC group and other groups (Fig. 3A). We then performed network analysis using the STRING database (confidence interaction score $\geq 0.400$ ) to extract biological meanings from the differentially expressed transcripts. The network analysis showed a highly clustered network (average local clustering coefficient: 0.555) containing 59 nodes with 169 edges (expected number of edges: 21), suggesting significantly more interaction than expected for a random set of genes of similar size ( $p$ value $\left.<1.0 \times 10^{-16}\right)$. The transcriptome was dominated by inflammation, cholesterol/steroid metabolism, and endoplasmic reticulum (ER) stress, as determined by k-means clustering (number of clusters $=3$, Fig. 3B). KEGG pathway analysis further confirmed that 7-KC regulated pathways related to inflammation (IL-17 signaling pathway, TNF signaling pathway, cytokine-cytokine receptor interaction, and NF-kappa B signaling pathway), metabolism (steroid biosynthesis and metabolic pathways), and ER stress (protein processing in endoplasmic reticulum), which included the genes encoding IkBa (Nfkbia), TNF-a (Tnf), binding immunoglobulin protein (BiP, Hspa5) and C/EBP homologous protein (CHOP, Ddit3) (Fig. 3C, Table S1 in the Data Supplement).

\section{ROS mediates 7-KC-induced proinflammatory responses}

In cultured macrophages, we confirmed that 7-KC significantly increased the expression of MCP-1 and tumor necrosis actor-a (TNF-a) (Fig. 4A). Moreover, 7-KC induced nuclear factor-kappa B (NF-KB) activation, as determined by monitoring p65 nuclear translocation (Fig. 4B). Previous reports have identified 7-KC-induced reactive oxygen species (ROS) production in rat PC-12 cells and mouse J774A.1 cells. ${ }^{32,33}$ Furthermore, RNA-Seq revealed that 7-KC regulated the expression of transcripts related to ER stress (Fig. 3B and C), which is regarded as the major inducer of mitochondrial ROS. ${ }^{34}$ Therefore, we hypothesized that mitochondrial ROS might play a key role in the mechanisms of 7-KC-induced proinflammatory responses. 7-KC increased ROS in the mitochondrial fraction compared to vehicle or cholesterol (Fig. 4C). N-acetyl-L-cysteine (NAC), a precursor of glutathione that acts as a ROS scavenger, abrogated 7-KC-induced ROS production (Fig. 4D) and TNF-a expression (Fig. 4E).

Although previous studies have reported that 7-KC increases proinflammatory cytokine levels through TLR4, ${ }^{35}$ 7-KC-induced proinflammatory responses were not decreased in peritoneal macrophages isolated from TLR4-deficient mice (Figure $S 4$ in the Data Supplement). Liver $X$ receptors (LXRs) $a$ and $\beta$ are recognized receptors for oxysterols, including 20(S)-, 22-, 24-, 25-, and 27-hydroxycholesterol and 24,25epoxycholesterol ${ }^{36,37}$, while few reports have shown that 7-KC is a weak ligand of LXRs. ${ }^{29,38}$ We confirmed that 7-KC increased the expression of LXR target genes related to lipid metabolism, such as ATP binding cassette subfamily A member 1 (ABCA1), ATP binding cassette subfamily G member 1 (ABCG1), and sterol regulatory element-binding protein 1c (SREBP-1c) (Figure S5 in the Data Supplement). Silencing LXRa and $\beta$ with small interfering RNA (siRNA), however, did not inhibit 7-KCinduced proinflammatory responses (Figure S6A and B in the Data Supplement). These results 
demonstrated that 7-KC induced monocyte/macrophage-mediated inflammation through mitochondrial ROS-mediated mechanisms but not through LXR or TLR4.

\section{7-KC induced ER stress and mitochondrial ROS}

ER stress is the major inducer of mitochondrial ROS. ${ }^{34}$ Based on our transcriptomic analysis (Fig. 3), ER stress was identified as one of the key signaling pathways in the 7KC-induced response. Western blotting showed that 7-KC significantly increased the levels of PKR-like endoplasmic reticulum kinase (PERK) and CHOP, molecules associated with ER stress (Fig. 5A, Figure S7 in the Data Supplement). Previous studies have demonstrated that U18666A, a blocker of free cholesterol transport from the plasma membrane to the ER, decreases cholesterol-induced ER stress. ${ }^{39}$ Thus, we examined the effect of U18666A on 7-KCinduced ER stress. The 7-KC-induced expression of molecules related to ER stress, production of mitochondrial ROS and expression of proinflammatory cytokines were significantly decreased by U18666A (Fig. 5B, C and D, Figure S8 in the Data Supplement). ER oxidase 1 a (ERO1-a), an important source of ROS during ER stress, ${ }^{40,41}$ was found to mediate 7-KC-induced proinflammatory cytokine expression in peritoneal macrophages transfected with PERK- or ER01-a-specific siRNA (Fig. 5E, Figure S9 in the Data Supplement).

\section{Discussion}

The present study investigated the effects of exogenous 7-KC in a mouse myocardial IR injury model and in cultured monocytes/macrophages. The novel findings included the following: 1) we created a murine model fed with 7-KC and underwent myocardial IR, 2) dietary 7-KC exacerbated myocardial IR injury through CCR2-positive monocyte/macrophage-mediated inflammation in mice, 3) 7-KC induced the differentiation and activation of monocytes/macrophages, and 4) ER stress and mitochondrial ROS production were involved in the inflammation observed in macrophages treated with 7-KC.

In our study, we reported the adverse effect of dietary 7-KC in the WT murine IR model for the first time. The plasma 7-KC level in our in vivo murine model fed with 7KWD reached at approximately $400 \mathrm{ng} / \mathrm{mL}$, which was similar to 7-KC levels in CAD patients, approximately 10 to $500 \mathrm{ng} / \mathrm{mL}$, who were not familial hypercholesterolemia in previous clinical studies. ${ }^{23,28}$ Therefore, results of our in vivo study are relevant to provide mechanistic insights into clinical observations that high 7-KC was associated with worse CAD outcomes.

In this study, we employed unbiased transcriptomic analysis and determined the molecular mechanisms of 7-KC-induced inflammation in monocytes/macrophages. Huang et al. previously reported that 7-KC induced inflammation in human retinal pigment epithelium through a TLR4-dependent pathway by using a TLR4 inhibitor. ${ }^{35}$ However, in our study, 7-KC increased proinflammatory cytokine levels in peritoneal macrophages isolated from TLR4-deficient mice, excluding a role for TLR4 in 7-KC-induced inflammation in macrophages. This difference may be because of the difference in cell type or the method of TLR4 inhibition. We revealed that 7-KC induced the expression of transcripts related to metabolic systems by 
RNA-Seq and confirmed that 7-KC increased the expression of LXR target genes such as ABCA1, ABCG1 and SREBP-1C. Although modulating LXRs might contribute to inflammatory gene expression by interfering with the LXR-mediated transrepression of inflammatory genes, ${ }^{42,43}$ we excluded this possibility through knockdown experiments targeting LXRa and $\beta$. Pedruzzi et al. reported that 7-KC induced NADPH oxidase 4 (NOX4) and ROS production in smooth muscle cells. ${ }^{19}$ Although 7-KC induced ROS production, RNA-Seq showed that the expression of NOX4 did not increase with 7-KC treatment in macrophages (data not shown).

ER stress is regarded as the major inducer of mitochondrial ROS, and ERO1-a regulated by CHOP plays an important role as a protein disulfide oxidase in ER stress-induced ROS production. ${ }^{40,41}$ ERO1-a mediates the transfer of electrons from protein disulfide isomerase to oxygen, resulting in ROS production. In addition, ERO1-a increases inositol-1,4,5-trisphosphate receptor-mediated $\mathrm{Ca}^{2+}$ release from the ER, and then the released $\mathrm{Ca}^{2+}$ accumulates in the mitochondria and disrupts the electron transport chain, leading to the production of mitochondrial ROS. We employed U18666A, a cholesterol transport inhibitor, and siRNA against PERK or ERO1- $\mathrm{a}$ and found that inhibition of ER stress decreased 7-KC-induced ROS production; thus, it seemed that 7-KC increased ROS production mainly through ER stress, not through NOX4 upregulation in macrophages.

In the current era of early reperfusion therapy, myocardial IR injury is a crucial and residual therapeutic target for reducing infarct size and improving long-term prognosis. No medical therapies, however, are currently available for the treatment of myocardial IR injury. We have shown that inflammation in the heart, which is primarily mediated by monocytes/macrophages, synergistically plays a role in IR injury, even in the absence of mitochondrial permeability transition pore opening. ${ }^{11,44}$ In the present study, CCR2 deficiency abrogated the effect of 7-KC on the infarct size after myocardial IR; therefore, we concluded that 7-KC exacerbated myocardial IR injury primarily through monocyte/macrophage-mediated inflammation. We also reported some findings related to inflammation: 1) 7-KC increased the expression of CCR2 in BMDMs, 2) 7-KC increased the chemotactic activity of macrophages, and 3) 7-KC increased the expression of MCP-1 and TNF- $a$. These findings suggest that 7-KC promotes macrophage accumulation in the IR heart by increasing MCP-1 and CCR2 expression on macrophages and that these macrophages release cytotoxic factors, such as TNF- $a$, resulting in a larger infarct size. Song et al. reported that a high plasma 7-KC level was related to a high rate of cardiovascular events, including $\mathrm{AMI},{ }^{28}$ which might result from 7-KC-induced chronic activation of circulating CCR2-positive monocytes. Based on our findings, 7-KC is a potential therapeutic target in patients with AMI, and targeting 7-KC may ameliorate myocardial inflammation and IR injury. We previously demonstrated that ezetimibe, a cholesterol transporter Niemann-pick C1-like 1 inhibitor, suppresses 7-KC absorption in the intestine and decreases the serum 7-KC concentration. ${ }^{25}$ Therefore, our findings could be translated into practice through clinical trials. Furthermore, because it has been reported that 7-KC is related to other cardiometabolic diseases, such as nonalcoholic fatty liver disease ${ }^{45}$ and age-related macular degeneration, ${ }^{46} 7-\mathrm{KC}$ could also be a therapeutic target in such diseases. It is noteworthy that the 7-KC 
level was significantly increased in heart tissue, not only in the blood (data not shown), and the related roles should be explored in other heart diseases.

There is a limitation to the present study. In vitro assays performed with 7-KC may not represent the activity of 7-KC in an in vivo environment, in which 7-KC is contained in lipoproteins such as low-density lipoprotein cholesterol in the circulating blood. Therefore, the kinetics of 7-KC, including interactions with the cell membrane and receptors, might be different in in vivo situations.

In conclusion, we showed for the first time that dietary 7-KC exacerbates myocardial IR injury through CCR2-positive macrophage-mediated inflammation. ER stress and oxidative stress are key processes involved in the 7-KC-induced proinflammatory responses in macrophages.

\section{Methods}

\section{Experimental animals}

Adult male C57BL/6J mice (10 weeks old) (Japan SLC Inc., Shizuoka, Japan), CCR2-deficient mice on the C57BL/6J background (10 weeks old) (CLEA Japan Inc., Tokyo, Japan), TLR4-deficient mice on the C57BL/6J background (10 weeks old) (Oriental Bio Service Inc., Kyoto, Japan) and CX3CR1-GFP/CCR2RFP mice on the C57BL/6J background (10 weeks old) (gifted by Dr. R. Yamasaki) were used in this study. ${ }^{31}$ All mice were maintained on a 12-hour light/dark cycle and fed either a normal laboratory diet or a high-fat high-cholesterol diet and water ad libitum. The study protocol was reviewed and approved by the Committee on the Ethics of Animal Experiments, Kyushu University Faculty of Medicine, which is compatible with the ARRIVE guidelines. All the experiments were conducted following the American Physiological Society guidelines and the NIH Guide for the Care and Use of Laboratory Animals, 8th edition.

\section{Measurement of plasma lipid levels}

Plasma was collected for cholesterol, oxysterol, and triglyceride measurements. Total cholesterol, lowdensity lipoprotein cholesterol, high-density lipoprotein cholesterol, and triglycerides were measured using enzymatic assay kits (FUJIFILM Wako Pure Chemical Co., Osaka, Japan). Lipids extracted plasma were saponified at room temperature overnight in the dark. Unsaponified lipids were applied to a Sep-Pak Vac silica cartridge (Waters Corporation, Milford, MA) to separate oxysterols and sterols. ${ }^{47}$ Oxysterols, including 7-KC, were converted into trimethylsilyl ethers in a mixture of trimethylchlorosilane, 1,1,1,3,3,3hexamethyldisilazane, and dried pyridine $(1: 3: 9, v: v: v)$ for 30 minutes at room temperature.

Oxysterols were measured by gas chromatography-mass spectrometry using a GCMS-QP2010 system (Shimadzu Co., Kyoto, Japan) equipped with an SPB-1-fused silica capillary column of $60 \mathrm{~m} \times 0.25 \mathrm{~mm}$ and $0.25-\mu \mathrm{m}$ phase thickness (Supelco Inc., Bellefonte, PA).

\section{Mouse myocardial IR injury model}


Mice were fed a normal laboratory diet (CRF-1), WD, or 7KWD for 3 weeks, and then surgery causing myocardial IR was performed. The WD contained $40 \%$ fat, $1 \%$ cholesterol and $0.5 \%$ cholic acid, and the 7KWD contained $40 \%$ fat, $0.9 \%$ cholesterol, $0.1 \%$ 7-ketocholesterol and $0.5 \%$ cholic acid. All these diets were purchased from ORIENTAL YEAST CO., Ltd. (Tokyo, Japan), and supplemental 7-KC was purchased from Sigma-Aldrich (St. Louis, MO). A murine model of myocardial IR injury was established as previously described. ${ }^{48,49}$ Mice were kept warm on a heating pad and anesthetized with $1.5 \%$ isoflurane in $2 \mathrm{~L} / \mathrm{min}$ oxygen. Subsequently, the mice were intubated and ventilated with a respirator. The heart was exposed by a left thoracotomy and pericardiotomy. The left anterior descending artery (LAD) was temporarily ligated with an $8-0$ nylon suture and silicon tube (1 mm OD) for 30 minutes, and then the silicon tube was removed to restart the blood flow.

\section{Preparation of primary macrophages}

As previously described, murine peritoneal and BMDMs were prepared for in vitro assays. ${ }^{50}$ Peritoneal macrophages were collected as described below. Brewer's thioglycolate medium (BD Diagnostic Systems, Sparks, MD) was injected into the murine peritoneal cavity 3 days before macrophage collection. Ice-cold phosphate-buffered saline (PBS) was injected into the peritoneal cavity, and cells were harvested. Red blood cells were lysed with ammonium-chloride-potassium lysis buffer (Thermo Fisher Scientific Inc., Cleveland, $\mathrm{OH}$ ). Then, the cells were washed with PBS, resuspended in Roswell Park Memorial Institute 1640 (RPMI-1640) medium (Sigma-Aldrich) supplemented with 10\% fetal bovine serum (FBS) (SigmaAldrich) and 1\% penicillin/streptomycin and plated in culture plates. One hour later, the nonadherent cells were removed, and the remaining adherent cells were cultured further in fresh medium. Bone marrowderived macrophages were harvested from the tibiae and femurs of donor mice. After red blood cell lysis and washing, the cells were resuspended and cultured in RPMI-1640 medium supplemented with 10\% FBS, $1 \%$ penicillin/streptomycin, and $20 \mathrm{ng} / \mathrm{mL}$ M-CSF (R\&D Systems, Minneapolis, MN).

\section{siRNA transfection}

ON-TARGETplus Non-targeting Control Pool or ON-TARGETplus SMARTPool siRNAs targeting mouse Nr1h3, Nr1h2, Eif2ak3, or Ero1/ were purchased from Horizon Discovery Ltd. (Cambridge, UK). All siRNAs were packaged in lipid nanoparticles (LNPs) and transfected into cells. Cultured cells were transfected with siRNA for 24 hours and then treated with cholesterol or 7-KC 12 hours after transfection was completed. siRNA-loaded LNPs were formulated as previously described. ${ }^{51}$ Briefly, $400 \mu \mathrm{L}$ of lipid solution containing $1.875 \mathrm{mM}$ ssPalm0-Phe (Product\# COATSOME® SS-OP, NOF CORPORATION, Tokyo, Japan), $450 \mu \mathrm{M}$ polyethylene glycol-dimyristoylglycerol (Product\# SUNBRIGHT® GM-020, NOF CORPORATION), and $1.875 \mathrm{mM}$ cholesterol (Sigma-Aldrich) in ethanol was prepared. Then, $10 \mu \mathrm{g}$ of siRNA in $124 \mu \mathrm{L}$ of $20 \mathrm{mM}$ malic acid buffer ( $\mathrm{pH} \mathrm{3.0)}$ was gradually added to the lipid solution under vigorous mixing. The mixed solution was diluted with $1 \mathrm{~mL}$ of malic acid buffer and then mixed vigorously with $3 \mathrm{~mL}$ of PBS. This diluted solution was subjected to ultrafiltration with Amicon Ultra-4 (Merck Millipore, Billerica, MA) two or more times. The encapsulation efficiency and recovery ratio of siRNA in this LNP solution were measured with a RiboGreen assay (Thermo Fisher Scientific Inc.) as previously described. ${ }^{52}$ 


\section{Quantification of myocardial infarction}

Twenty-four hours after reperfusion, mice were anesthetized with $1.5 \%$ isoflurane in $2 \mathrm{~L} / \mathrm{min}$ oxygen and intubated. After ligating the LAD again, 2\% Evans blue dye (Sigma-Aldrich) was injected into the inferior vena cava to dye the nonischemic area of the heart. Hearts were harvested and washed by saline perfusion. The harvested hearts were frozen in liquid nitrogen and cut into sequential cross-sections at a thickness of $1 \mathrm{~mm}$. The sections were incubated with 1.5\% 2,3,5-triphenyltetrazolium chloride (TTC) (Sigma-Aldrich) for 20 minutes at $37^{\circ} \mathrm{C}$. After dyeing with TTC, the sections were placed in $10 \%$ formaldehyde overnight. Then, the sections were photographed under a microscope. The infarcted area (TTC negative/Evans blue negative, white), the noninfarcted area within the area at risk (AAR) (TTC positive/Evans blue negative, red), the nonischemic area (TTC positive/Evans blue positive, purple), and the AAR (Evans blue negative, red and white) were quantified using ImageJ software (version $1.51 \mathrm{~h}$ ).

\section{Immunohistochemistry}

Paraffin sections of the heart were prepared for immunohistochemistry. Twenty-four hours after reperfusion, hearts were harvested, fixed in $10 \%$ formaldehyde for 24 hours, and then embedded in paraffin. Serial cross-sections of the heart cut at a thickness of $5 \mu \mathrm{m}$ at the midventricular level were used for analysis. After blocking with $2.5 \%$ goat serum (ImmPRESS, Goat Anti-Rat IgG Polymer Kit, Peroxidase, Vector Laboratories, Burlingame, $C A$ ), the sections were subjected to immunostaining for macrophages using an anti-mouse Mac-3 antibody (BD Pharmingen, San Diego, CA). After an overnight incubation with the primary antibody at $4^{\circ} \mathrm{C}$, the sections were washed 3 times with PBS and incubated with peroxidaseconjugated anti-rat IgG at room temperature for 30 minutes. Then, the sections were washed 3 times with PBS and incubated with a peroxidase substrate (ImmPACT, AMEC Red Peroxidase Substrate Kit, Vector Laboratories) for 10 minutes. After counterstaining with hematoxylin, the sections were imaged with a stereomicroscope. Mac-3-positive cells were counted using ImageJ software (version 1.51h).

\section{Flow cytometry}

Twenty-four hours after reperfusion, hearts were harvested, cut into small pieces, and digested with a cocktail of $450 \mathrm{U} / \mathrm{mL}$ collagenase type I, $125 \mathrm{U} / \mathrm{mL}$ collagenase type $\mathrm{XI}, 60 \mathrm{U} / \mathrm{mL}$ DNase I, and $60 \mathrm{U} / \mathrm{mL}$ hyaluronidase (Sigma-Aldrich) in PBS containing $20 \mathrm{mM} \mathrm{HEPES}$ at $37^{\circ} \mathrm{C}$ for 40 minutes. The cell suspensions were washed with PBS, and Fc receptors were blocked with anti-CD16/32 antibodies (BD Pharmingen). After blocking, the cell suspensions were incubated with a cocktail of antibodies against leukocytes (anti-CD45-PE-Cy7), myeloid cells (anti-CD11b-APC-Cy7), B cells (anti-B220-PE), T cells (antiCD90.2-PE), NK cells (anti-CD49b-PE, NK1.1-PE), granulocytes (anti-Ly-6G-PE), monocyte subsets (anti-Ly6C-FITC), and macrophages (anti-F4/80-Alexa Fluor 647) (BD Pharmingen) for 1 hour at $4^{\circ} \mathrm{C}$. 7-AAD (BD Pharmingen) was applied to exclude nonviable cells just before starting the analysis. The stained cells were analyzed with a Gallios flow cytometer (Beckman Coulter Inc., CA). Monocytes/macrophages were identified as CD45 + CD11b + Lineage (B220/CD90.2/CD49b/NK1.1/Ly-6G)- Ly-6Chigh/low cells.

\section{Quantitative real-time PCR}


RNA was extracted from cultured macrophages with an Illustra RNA Spin Mini Kit (GE Healthcare, Chicago, IL), and cDNA was synthesized with a PrimeScript RT Reagent Kit (TAKARA BIO Inc., Shiga, Japan) according to the manufacturer's instructions. Real-time PCR was performed with TB Green Premix Ex Taq II (TAKARA BIO Inc.) and a StepOnePlus Real-time PCR System (Applied Biosystems, Waltham, MA). The primer sequences are listed in Table $S 2$ in the Data Supplement.

\section{Evaluation of CX3CR1 and CCR2 expression}

Bone marrow cells harvested from CX3CR1-GFP CCR2-RFP mice were cultured for 5 days and then pretreated with $10 \mu \mathrm{M}$ cholesterol or 7-KC for 24 hours before stimulation with $10 \mathrm{ng} / \mathrm{mL}$ lipopolysaccharide (LPS) and $10 \mathrm{ng} / \mathrm{mL}$ interferon- $\gamma$ (IFN- $\gamma$ ) for 24 hours to induce differentiation into inflammatory macrophages. After nuclear counterstaining (Hoechst 33342, Thermo Fisher Scientific Inc.), CX3CR1-positive cells (GFP-positive) and CCR2-positive cells (RFP-positive) were observed under a confocal laser scanning microscope (FV1000, Olympus, Tokyo, Japan).

\section{Chemotaxis assay}

The chemotactic activity of macrophages was measured in a 96-well chemotaxis chamber with a 5- $\mu \mathrm{m}$ pore-sized membrane (ChemoTx, Neuro Probe Inc., Gaithersburg, MD) according to the manufacturer's instructions. MCP-1 in RPMI-1640 medium was added to the lower chambers, and peritoneal macrophages harvested from wild-type (WT) mice fed the WD or 7KWD for 3 weeks ( $1 \times 10^{7}$ cells per $\mathrm{mL}$ ) were placed in the upper chambers. After a 4 -hour incubation at $37^{\circ} \mathrm{C}$, the upper surface of the membrane was washed with PBS, and $20 \mathrm{mM}$ ethylenediaminetetraacetic acid (EDTA) was applied. After a 20minute incubation at $4^{\circ} \mathrm{C}$, the added EDTA was removed and centrifuged. The migrated cells in the lower chambers were counted.

\section{RNA sequencing}

Peritoneal macrophages were stimulated with $10 \mu \mathrm{M}$ cholesterol or 7-KC for 24 hours, and then RNA was extracted with ISOGEN (Nippon Gene, Tokyo, Japan). RNA-Seq was performed using an Illumina NextSeq 500 (Illumina Inc., Tokyo, Japan). Among all detected genes, differentially expressed transcripts between the cholesterol group and the 7-KC group were chosen according to the stated criteria (fold change $>2$ or $<0.5$ and FDR $<0.05$ ). The obtained data were analyzed using STRING software (version 11.5) and iDEP software (version 0.94).

\section{NF-KB activity assay}

NF-kB activity was evaluated by measuring p65 translocation into the nucleus using a TransAM NF-KB p65 Kit (Active Motif, Carlsbad, CA) according to the manufacturer's instructions. Nuclear extracts were extracted from cultured peritoneal macrophages, and protein concentrations were determined with a Bradford-based assay (Pierce Coomassie Plus Assay Kit, Thermo Fisher Scientific Inc.). The nuclear extracts were added to a 96-well plate coated with an oligonucleotide containing the NF-KB consensus sequence (5'-GGGACTTTTCC-3') and incubated for 1 hour at room temperature. An anti-NF-KB antibody was added to each well, and the plate was incubated for 1 hour at room temperature. After the plate was 
incubated for 1 hour with a horseradish peroxidase-conjugated secondary antibody, specific binding was detected with a microplate spectrophotometer based on the absorption at $450 \mathrm{~nm}$ with a reference wavelength of $655 \mathrm{~nm}$.

\section{Measurement of mitochondrial ROS}

Mitochondrial superoxide was observed and quantified using the mitochondrial superoxide indicator MitoSOX Red (Thermo Fisher Scientific Inc.). After peritoneal macrophages were stimulated with $10 \mu \mathrm{M} 7$ $\mathrm{KC}$ for 24 hours, the cells were incubated with a $2.5 \mu \mathrm{M}$ MitoSOX solution. After nuclear counterstaining, the fluorescence intensity of MitoSOX was quantified using an FV1000.

\section{Western blotting}

Total protein was extracted from peritoneal macrophages using Cell Lysis Buffer (Cell Signaling Technology Inc., Danvers, MA) according to the manufacturer's instructions. Protein concentrations were determined with a BCA assay (Pierce BCA Protein Assay Kit, Thermo Fish Scientific Inc.). Proteins were separated on 4-20\% gradient SDS/polyacrylamide gels and blotted onto PVDF membranes. The following antibodies were used as primary antibodies: anti-PERK (1:2000), anti-BiP (1:2000), anti-CHOP (1:2000) (Cell Signaling Technology Inc.), and anti- $\beta$-actin (1:10000, Sigma-Aldrich). Anti-Rabbit IgG, HRP-linked whole Ab donkey (GE Healthcare) was used as secondary antibody (1:5000).

Chemiluminescence images were acquire with an Image Quant LAS-4010 (GE Healthcare). Densitometric analyses were performed using an Image Quant TL (GE Healthcare).

\section{Statistical analysis}

Data are expressed as the mean \pm SEM. Statistical analyses of the differences between two groups were performed using an unpaired t test, and analyses of the differences among three or more groups were performed by one-way or two-way ANOVA, followed by Tukey's post-hoc multiple comparison test using Prism Software version 8.0 (GraphPad Software, San Diego, CA). Statistical analyses of flow cytometry data were performed using the chi-square test. $P$ values less than 0.05 were considered statistically significant.

\section{Declarations}

\section{Acknowledgments}

We thank Kozue Nakamura for her excellent technical assistance. We thank NOF CORPORATION for providing materials for LNP formulation.

\section{Author contributions}

T.U., T.M., S.K., J.K. and H.T. designed the study and prepared the manuscript. T.U., T.K. and I.B. acquired and analyzed experimental data. T.U., Y.H. and R.Y. performed experiments using CX3CR1-GFP/CCR2-RFP 
mice. I.I. measured of murine plasma oxysterols level. T.U., I.B. and H.A. prepared LNP. All authors have approved the final version of the manuscript.

\section{Data availability.}

All RNA sequencing data are available at NCBI Sequence Read Archive (SRA) with the project accession number PRJNA810596.

\section{Funding}

This work was supported by JSPS KAKENHI grant number $21 \mathrm{H} 02916$ to T.M. and by JST, CREST grant number 17941843 to T.M.

\section{Additional information}

TM has received personal fees (Abbott, Bayer Yakuhin, and MSD) and research funding (Kowa, Amgen and Bayer Yakuhin) outside the submitted work. HT has received lecture fees (Kowa, Teijin Pharma, Nippon Boehringer Ingelheim, Mitsubishi Tanabe Pharma, Pfizer Japan, Ono Pharmaceutical, Daiichi Sankyo, Novartis Pharma, Bayer Yakuhin, Otsuka Pharmaceuticalm, and AstraZeneca), manuscript fees (Nippon Rinsho), research funding (Mitsubishi Tanabe Pharma, Nippon Boehringer Ingelheim, IQVIA Services Japan, MEDINET, Medical Innovation Kyushu, Kowa, Daiichi Sankyo, Johnson \& Johnson, and NEC Corporation) and scholarship funds (Abbott Medical Japan, Otsuka Pharmaceutical, Boston Scientific Japan, Ono Pharmaceutical, Bayer Yakuhin, Nippon Boehringer Ingelheim, St. Mary's Hospital, Teijin Pharma, Daiichi Sankyo, and Mitsubishi Tanabe Pharma) outside the submitted work. The other authors report no conflicts.

\section{References}

1. Schmidt, M., Jacobsen, J. B., Lash, T. L., Botker, H. E. \& Sorensen, H. T. 25 year trends in first time hospitalisation for acute myocardial infarction, subsequent short and long term mortality, and the prognostic impact of sex and comorbidity: a Danish nationwide cohort study. BMJ 344, e356, doi:10.1136/bmj.e356 (2012).

2. Hellermann, J. P. et al. Heart failure after myocardial infarction: prevalence of preserved left ventricular systolic function in the community. Am. Heart J. 145, 742-748, doi:10.1067/mhj.2003.187 (2003).

3. Goldberg, R. J. et al. Six-month outcomes in a multinational registry of patients hospitalized with an acute coronary syndrome (the Global Registry of Acute Coronary Events [GRACE]). Am. J. Cardiol. 93, 288-293, doi:10.1016/j.amjcard.2003.10.006 (2004).

4. Degano, I. R. et al. Twenty-five-year trends in myocardial infarction attack and mortality rates, and case-fatality, in six European populations. Heart 101, 1413-1421, doi:10.1136/heartjnl-2014-307310 (2015). 
5. Menees, D. S. et al. Door-to-balloon time and mortality among patients undergoing primary PCI. N. Engl. J. Med. 369, 901-909, doi:10.1056/NEJMoa1208200 (2013).

6. Yellon, D. M. \& Hausenloy, D. J. Myocardial Reperfusion Injury. N. Engl. J. Med. 357, 1121-1135 (2007).

7. Hausenloy, D. J. \& Yellon, D. M. Myocardial ischemia-reperfusion injury: a neglected therapeutic target. J. Clin. Invest. 123, 92-100, doi:10.1172/JCl62874 (2013).

8. Frantz, S. \& Nahrendorf, M. Cardiac macrophages and their role in ischaemic heart disease. Cardiovasc. Res. 102, 240-248, doi:10.1093/cvr/cvu025 (2014).

9. Peet, C., Ivetic, A., Bromage, D. I. \& Shah, A. M. Cardiac monocytes and macrophages after myocardial infarction. Cardiovasc. Res. 116, 1101-1112, doi:10.1093/cvr/cvz336 (2020).

10. Prabhu, S. D. It takes two to tango: monocyte and macrophage duality in the infarcted heart. Circ. Res. 114, 1558-1560, doi:10.1161/CIRCRESAHA.114.303933 (2014).

11. Nakano, Y. et al. Nanoparticle-Mediated Delivery of Irbesartan Induces Cardioprotection from Myocardial Ischemia-Reperfusion Injury by Antagonizing Monocyte-Mediated Inflammation. Sci. Rep. 6, 29601, doi:10.1038/srep29601 (2016).

12. Tokutome, M. et al. Peroxisome proliferator-activated receptor-gamma targeting nanomedicine promotes cardiac healing after acute myocardial infarction by skewing monocyte/macrophage polarization in preclinical animal models. Cardiovasc. Res. 115, 419-431, doi:10.1093/cvr/cvy200 (2019).

13. Fujiwara, M. et al. Nanoparticle incorporating Toll-like receptor 4 inhibitor attenuates myocardial ischaemia-reperfusion injury by inhibiting monocyte-mediated inflammation in mice. Cardiovasc. Res. 115, 1244-1255, doi:10.1093/cvr/cvz066 (2019).

14. Cannon, C. P. et al. Ezetimibe Added to Statin Therapy after Acute Coronary Syndromes. N. Engl. J. Med. 372, 2387-2397, doi:10.1056/NEJMoa1410489 (2015).

15. Sabatine, M. S. et al. Evolocumab and Clinical Outcomes in Patients with Cardiovascular Disease. N. Engl. J. Med. 376, 1713-1722, doi:10.1056/NEJMoa1615664 (2017).

16. LaRosa, J. C. et al. Intensive Lipid Lowering with Atorvastatin in Patients with Stable Coronary Disease. N. Engl. J. Med. 352, 1425-1435 (2005).

17. Gargiulo, S., Gamba, P., Testa, G., Leonarduzzi, G. \& Poli, G. The role of oxysterols in vascular ageing. J. Physiol. 594, 2095-2113, doi:10.1113/JP271168 (2016).

18. Gargiulo, S. et al. Plaque oxysterols induce unbalanced up-regulation of matrix metalloproteinase-9 in macrophagic cells through redox-sensitive signaling pathways: Implications regarding the vulnerability of atherosclerotic lesions. Free Radic. Biol. Med. 51, 844-855, doi:10.1016/j.freeradbiomed.2011.05.030 (2011).

19. Pedruzzi, E. et al. NAD(P)H oxidase Nox-4 mediates 7-ketocholesterol-induced endoplasmic reticulum stress and apoptosis in human aortic smooth muscle cells. Mol. Cell. Biol. 24, 10703-10717, doi:10.1128/MCB.24.24.10703-10717.2004 (2004). 
20. Ferre, N. et al. Increased susceptibility to exacerbated liver injury in hypercholesterolemic ApoEdeficient mice: potential involvement of oxysterols. Am. J. Physiol. Gastrointest. Liver Physiol. 296, G553-562, doi:10.1152/ajpgi.00547.2007 (2009).

21. Evans, T. D., Sergin, I., Zhang, X. \& Razani, B. Modulating Oxysterol Sensing to Control Macrophage Apoptosis and Atherosclerosis. Circ. Res. 119, 1258-1261, doi:10.1161/CIRCRESAHA.116.310155 (2016).

22. Sato, K. et al. Dietary cholesterol oxidation products accelerate plaque destabilization and rupture associated with monocyte infiltration/activation via the MCP-1-CCR2 pathway in mouse brachiocephalic arteries: therapeutic effects of ezetimibe. J Atheroscler Thromb 19, 986-998 (2012).

23. Takase, S. et al. Ezetimibe in Combination With Statins Ameliorates Endothelial Dysfunction in Coronary Arteries After Stenting: The CuVIC Trial (Effect of Cholesterol Absorption Inhibitor Usage on Target Vessel Dysfunction After Coronary Stenting), a Multicenter Randomized Controlled Trial. Arterioscler. Thromb. Vasc. Biol. 37, 350-358, doi:10.1161/ATVBAHA.116.308388 (2017).

24. Mutemberezi, V., Guillemot-Legris, O. \& Muccioli, G. G. Oxysterols: From cholesterol metabolites to key mediators. Prog. Lipid Res. 64, 152-169, doi:10.1016/j.plipres.2016.09.002 (2016).

25. Honda, K. et al. Lipid-Lowering Therapy With Ezetimibe Decreases Spontaneous Atherothrombotic Occlusions in a Rabbit Model of Plaque Erosion: A Role of Serum Oxysterols. Arterioscler. Thromb. Vasc. Biol. 38, 757-771, doi:10.1161/ATVBAHA.117.310244 (2018).

26. Brown, A. J., Dean, R. T. \& Jessup, W. Free and esterified oxysterol: formation during copper-oxidation of low density lipoprotein and uptake by macrophages. J. Lipid Res. 37, 320-335 (1996).

27. Oh, M. J. et al. Oxidized LDL signals through Rho-GTPase to induce endothelial cell stiffening and promote capillary formation. J. Lipid Res. 57, 791-808, doi:10.1194/jlr.M062539 (2016).

28. Song, J. et al. Association of Plasma 7-Ketocholesterol With Cardiovascular Outcomes and Total Mortality in Patients With Coronary Artery Disease. Circ. Res. 120, 1622-1631, doi:10.1161/CIRCRESAHA.117.311049 (2017).

29. Anderson, A. et al. 7-Ketocholesterol in disease and aging. Redox Biol 29, 101380, doi:10.1016/j.redox.2019.101380 (2020).

30. Schweizer, R. A., Zurcher, M., Balazs, Z., Dick, B. \& Odermatt, A. Rapid hepatic metabolism of 7ketocholesterol by 11 beta-hydroxysteroid dehydrogenase type 1: species-specific differences between the rat, human, and hamster enzyme. J. Biol. Chem. 279, 18415-18424, doi:10.1074/jbc.M313615200 (2004).

31. Yamasaki, R. et al. Differential roles of microglia and monocytes in the inflamed central nervous system. J. Exp. Med. 211, 1533-1549, doi:10.1084/jem.20132477 (2014).

32. Jang, E. R. \& Lee, C. S. 7-ketocholesterol induces apoptosis in differentiated PC12 cells via reactive oxygen species-dependent activation of NF-kappaB and Akt pathways. Neurochem. Int. 58, 52-59, doi:10.1016/j.neuint.2010.10.012 (2011).

33. Leonarduzzi, G., Biasi, F., Chiarpotto, E. \& Poli, G. Trojan horse-like behavior of a biologically representative mixture of oxysterols. Mol. Aspects Med. 25, 155-167, 
doi:10.1016/j.mam.2004.02.016 (2004).

34. Cao, S. S. \& Kaufman, R. J. Endoplasmic reticulum stress and oxidative stress in cell fate decision and human disease. Antioxid Redox Signal 21, 396-413, doi:10.1089/ars.2014.5851 (2014).

35. Huang, J. D., Amaral, J., Lee, J. W. \& Rodriguez, I. R. 7-Ketocholesterol-induced inflammation signals mostly through the TLR4 receptor both in vitro and in vivo. PLoS One 9, e100985, doi:10.1371/journal.pone.0100985 (2014).

36. Traversari, C., Sozzani, S., Steffensen, K. R. \& Russo, V. LXR-dependent and -independent effects of oxysterols on immunity and tumor growth. Eur. J. Immunol. 44, 1896-1903, doi:10.1002/eji.201344292 (2014).

37. Ducheix, S., Lobaccaro, J. M., Martin, P. G. \& Guillou, H. Liver X Receptor: an oxysterol sensor and a major player in the control of lipogenesis. Chem. Phys. Lipids 164, 500-514, doi:10.1016/j.chemphyslip.2011.06.004 (2011).

38. Janowski, B. A. et al. Structural requirements of ligands for the oxysterol liver $X$ receptors $L X R a$ and LXRß. Proc Natl Acad Sci U S A. 96, 266-271 (1999).

39. Chattopadhyay, A. et al. Cholesterol-Induced Phenotypic Modulation of Smooth Muscle Cells to Macrophage/Fibroblast-like Cells Is Driven by an Unfolded Protein Response. Arterioscler. Thromb. Vasc. Biol. 41, 302-316, doi:10.1161/ATVBAHA.120.315164 (2021).

40. Li, G. et al. Role of ERO1-alpha-mediated stimulation of inositol 1,4,5-triphosphate receptor activity in endoplasmic reticulum stress-induced apoptosis. J. Cell Biol. 186, 783-792, doi:10.1083/jcb.200904060 (2009).

41. Malhotra, J. D. \& Kaufman, R. J. Endoplasmic reticulum stress and oxidative stress: a vicious cycle or a double-edged sword? Antioxid Redox Signal 9, 2277-2293, doi:10.1089/ars.2007.1782 (2007).

42. Walcher, D. et al. LXR activation reduces proinflammatory cytokine expression in human CD4-positive lymphocytes. Arterioscler. Thromb. Vasc. Biol. 26, 1022-1028, doi:10.1161/01.ATV.0000210278.67076.8f (2006).

43. Gao, M. et al. Activation of liver $X$ receptor attenuates endothelin-1 expression in vascular endothelial cells. Int. J. Biochem. Cell Biol. 44, 2299-2307, doi:10.1016/j.biocel.2012.09.010 (2012).

44. Ikeda, G. et al. Nanoparticle-Mediated Simultaneous Targeting of Mitochondrial Injury and Inflammation Attenuates Myocardial Ischemia-Reperfusion Injury. J Am Heart Assoc 10, e019521, doi:10.1161/JAHA.120.019521 (2021).

45. Chang, J. et al. Dietary Oxysterol, 7-Ketocholesterol Accelerates Hepatic Lipid Accumulation and Macrophage Infiltration in Obese Mice. Front. Endocrinol. (Lausanne) 11, 614692, doi:10.3389/fendo.2020.614692 (2020).

46. Indaram, M. et al. 7-Ketocholesterol increases retinal microglial migration, activation, and angiogenicity: a potential pathogenic mechanism underlying age-related macular degeneration. Sci. Rep. 5, 9144, doi:10.1038/srep09144 (2015).

47. Dzeletovic, S., Breuer, O., Lund, E. \& Diczfalusy, U. Determination of cholesterol oxidation products in human plasma by isotope dilution-mass spectrometry. Anal. Biochem. 225, 73-80 (1995). 
48. Ikeda, G. et al. Nanoparticle-Mediated Targeting of Cyclosporine A Enhances Cardioprotection Against Ischemia-Reperfusion Injury Through Inhibition of Mitochondrial Permeability Transition Pore Opening. Sci. Rep. 6, 20467, doi:10.1038/srep20467 (2016).

49. Frankenreiter, S. et al. Cardioprotection by ischemic postconditioning and cyclic guanosine monophosphate-elevating agents involves cardiomyocyte nitric oxide-sensitive guanylyl cyclase. Cardiovasc. Res. 114, 822-829, doi:10.1093/cvr/cvy039 (2018).

50. Nakashiro, S. et al. Pioglitazone-Incorporated Nanoparticles Prevent Plaque Destabilization and Rupture by Regulating Monocyte/Macrophage Differentiation in ApoE-/- Mice. Arterioscler. Thromb. Vasc. Biol. 36, 491-500, doi:10.1161/ATVBAHA.115.307057 (2016).

51. Cho, R. et al. Silencing of VEGFR2 by RGD-Modified Lipid Nanoparticles Enhanced the Efficacy of Anti-PD-1 Antibody by Accelerating Vascular Normalization and Infiltration of T Cells in Tumors. Cancers (Basel) 12, doi:10.3390/cancers12123630 (2020).

52. Sato, Y. et al. A pH-sensitive cationic lipid facilitates the delivery of liposomal siRNA and gene silencing activity in vitro and in vivo. J. Control. Release 163, 267-276, doi:10.1016/j.jconrel.2012.09.009 (2012).

\section{Figures}


A

ND

WD

7KWD
B

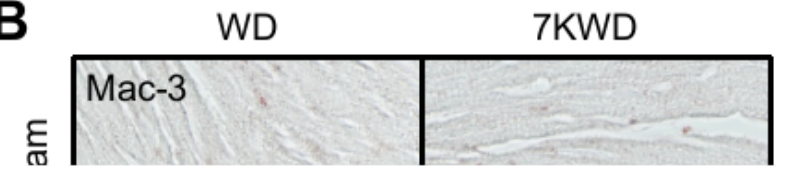

\section{Figure 1}

Dietary 7-KC increased the infarct size after myocardial IR through monocyte/macrophage-mediated inflammation. A, Infarct size at 24 hours after IR. Scale bar, $1 \mathrm{~mm}$. N=8-10. B, Macrophages (Mac-3positive cells) in the heart at 24 hours after IR. Scale bar, $20 \mu \mathrm{m}$. N=7. C, Percentages of Ly-6C-high or Ly$6 \mathrm{C}$-low monocytes/macrophages in the heart at 24 hours after IR assessed by flow cytometry. $\mathrm{N}=6 . \mathrm{D}$, Infarct size in CCR2-deficient mice at 24 hours after IR. N=6. ND, normal laboratory diet; WD, Western diet; 
7KWD, WD containing 7-KC; AAR, area at risk; IR, ischemia-reperfusion; Lin, lineage; Mo, monocyte; Mph, macrophage.

A

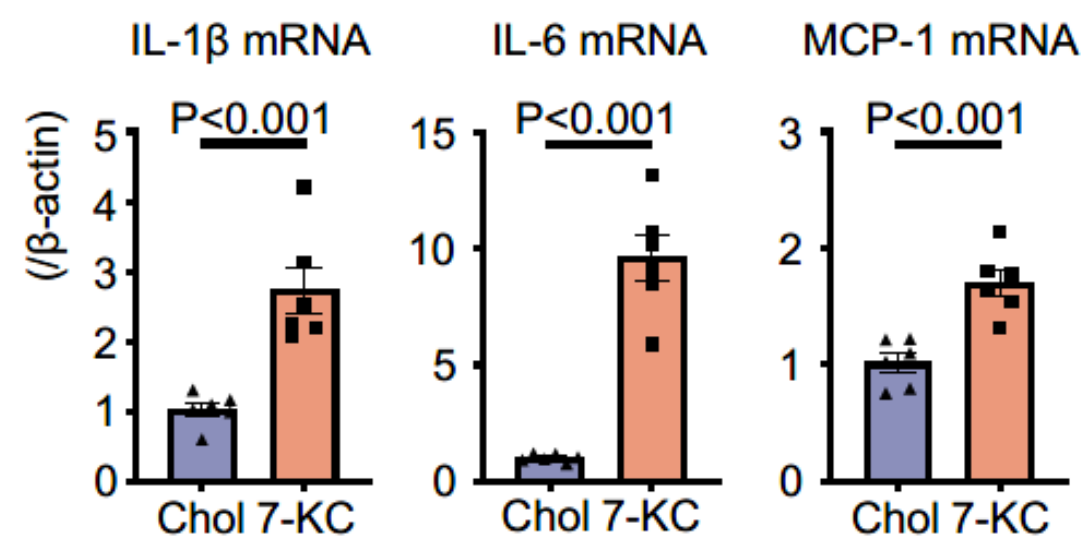

B
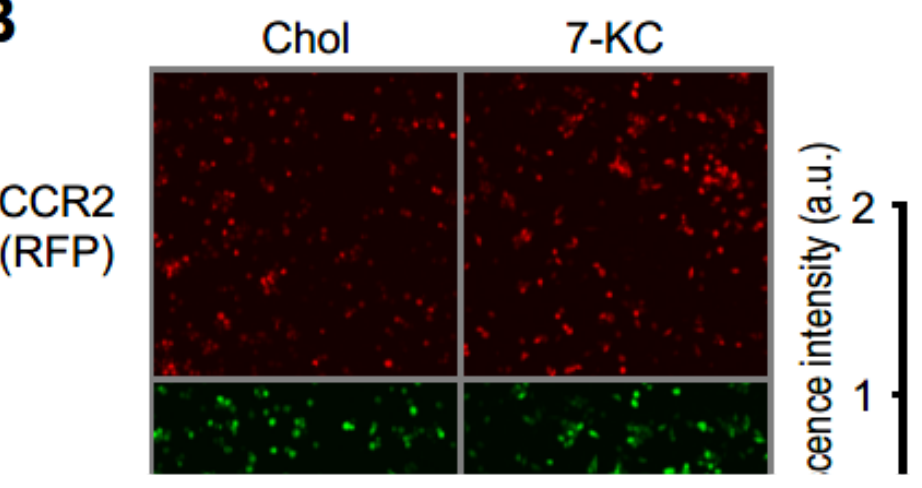

CCR2
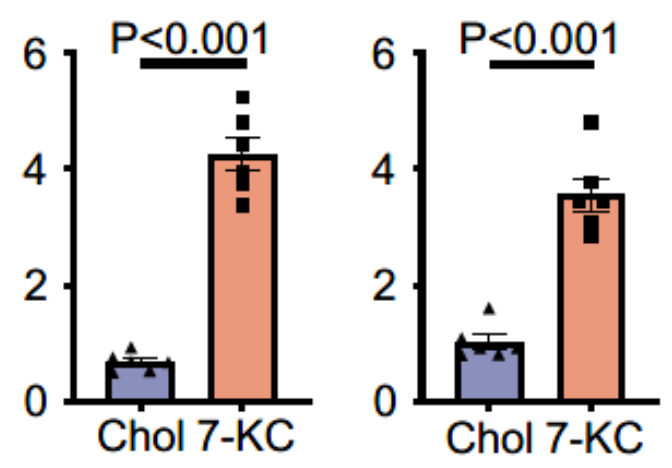

C

Migrated Macrophage

$P<0.01$

$P<0.001$

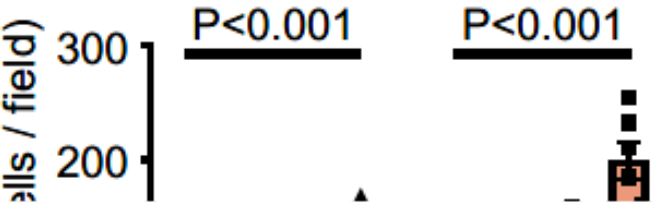

Figure 2 
7-KC induced a phenotypic shift toward inflammatory macrophages and increased the chemotactic function of macrophages. $A$ and $B$, Bone marrow-derived cells were pretreated with $10 \mu \mathrm{M}$ cholesterol or 7-KC for 24 hours and then stimulated with $10 \mathrm{ng} / \mathrm{mL}$ LPS and $10 \mathrm{ng} / \mathrm{mL}$ IFN- $\gamma$ for 24 hours. (A) mRNA expression of cytokines and iNOS in WT mice. $\mathrm{N}=6$. (B) CCR2 and CX3CR1 expression in CX3CR1GFP/CCR2-RFP mice. Scale bar, $100 \mu \mathrm{m}$. N=9. C, Chemotactic activity of peritoneal macrophages harvested from WT mice fed a WD or 7KWD for 3 weeks in response to MCP-1. N=6. P values were obtained by two-way ANOVA. Chol, cholesterol; 7-KC, 7-ketocholesterol; WD, Western diet; 7KWD, WD containing 7-KC; LPS, lipopolysaccharide; IFN-y, interferon-ץ. 
A
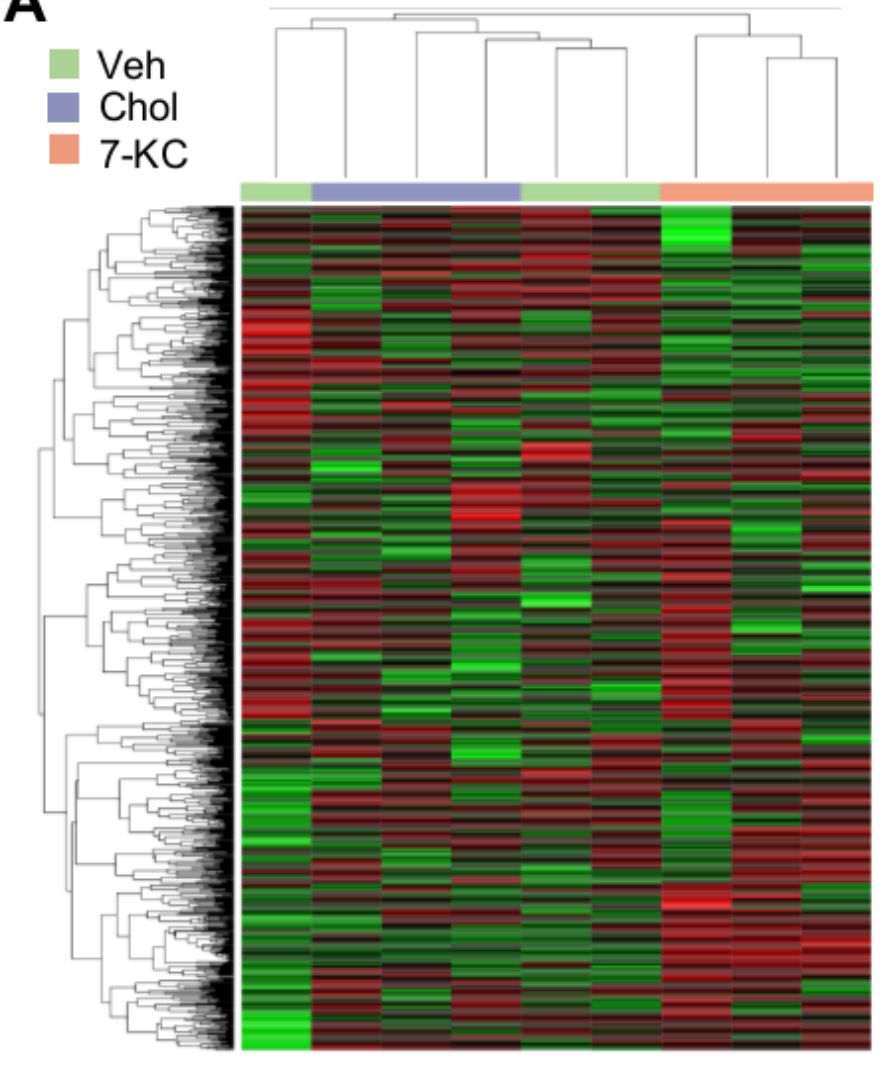

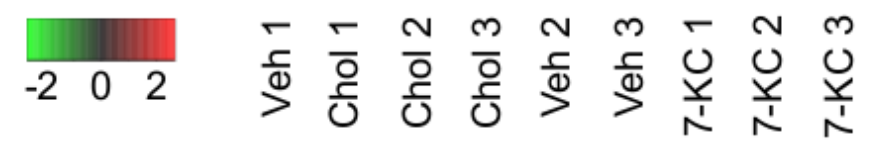

B

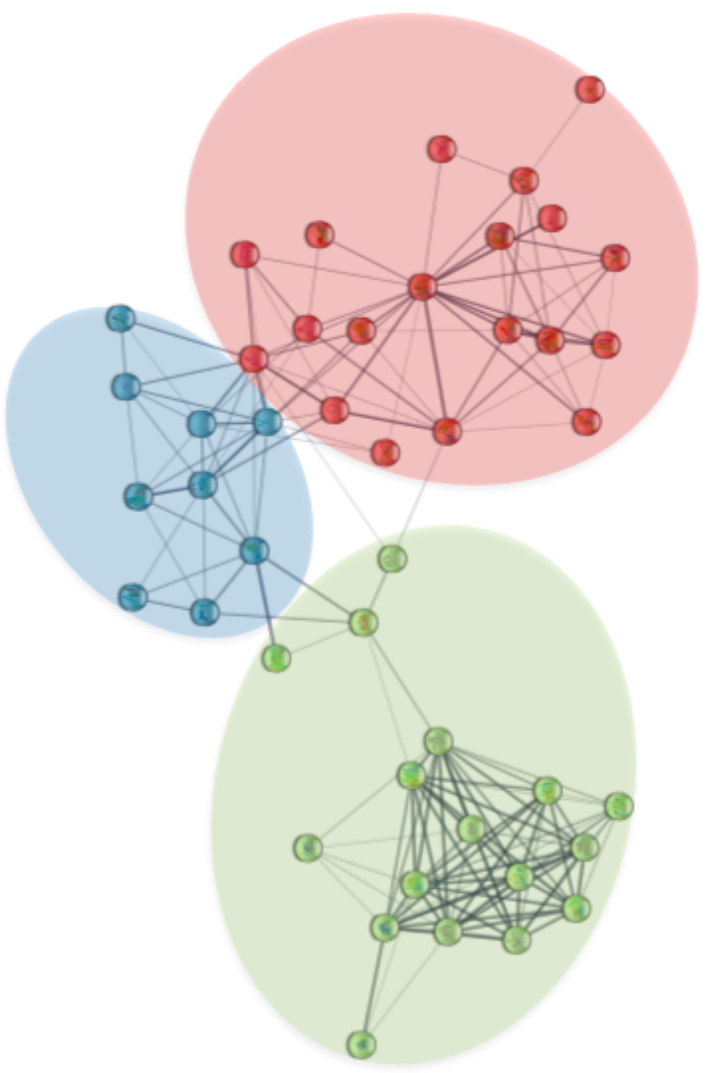

- Inflammation

Cholesterol/Steroid

- ER stress

C

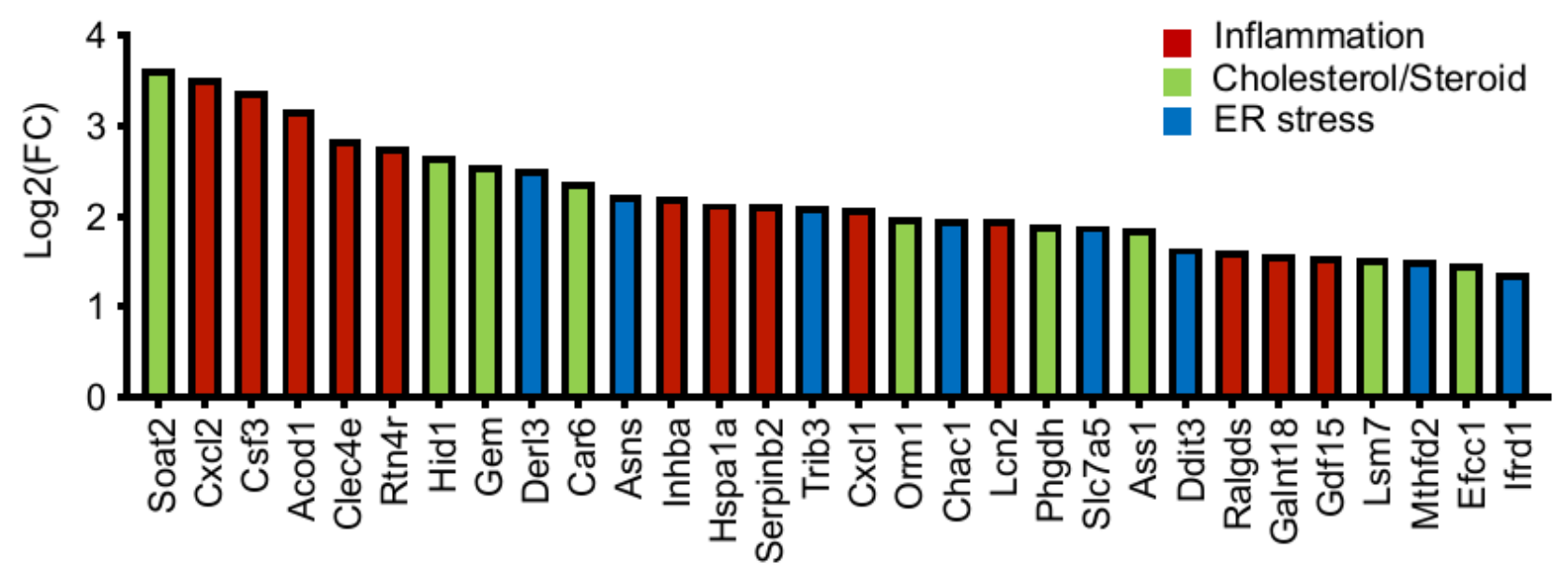

Figure 3

RNA-Seq revealed that 7-KC regulated the expression of transcripts related to inflammation, cholesterol biosynthesis and ER stress. RNA-Seq was performed using RNAs extracted from peritoneal macrophages treated with vehicle, $10 \mu \mathrm{M}$ cholesterol or 7-KC for 24 hours. N=3. A, Transcriptomic heatmap for the 3 groups. B and C, Network analysis (B) and top 30 differentially overexpressed mRNAs (C) in macrophages treated with 7-KC compared to those treated with cholesterol. Transcripts were categorized 
as inflammation- (red), cholesterol/steroid metabolism- (green), and ER stress-associated genes (blue). RNA-Seq, RNA sequencing; Veh, vehicle; Chol, cholesterol; 7-KC, 7-ketocholesterol; ER, endoplasmic reticulum; FC, fold change.

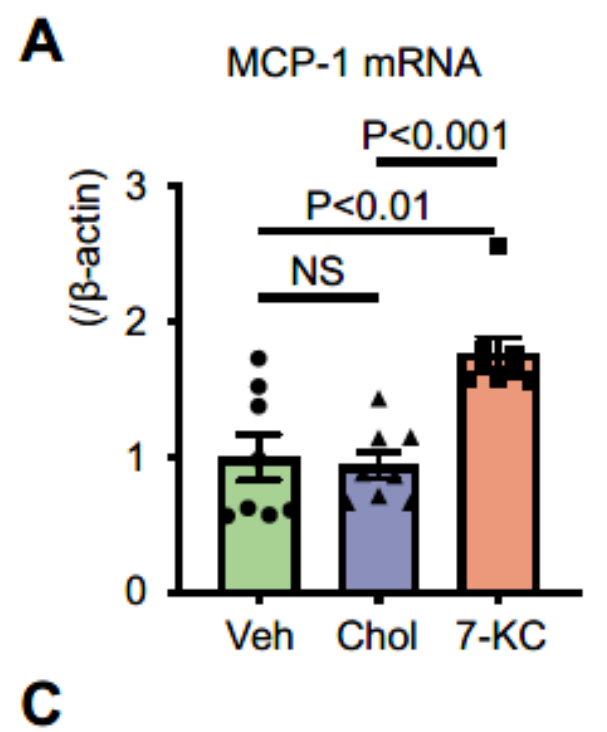

\section{Chol}

TNF- $\alpha$ mRNA

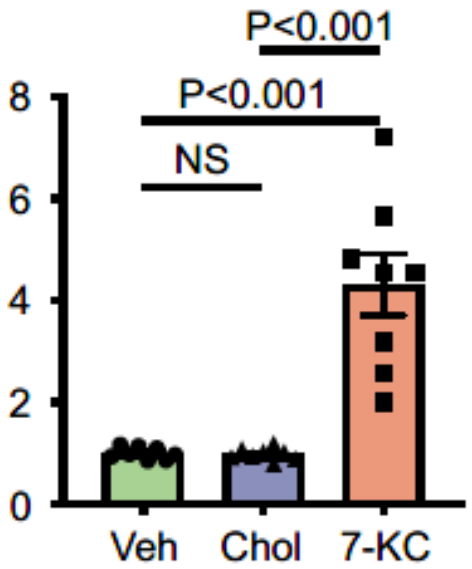

B NF-KB activity

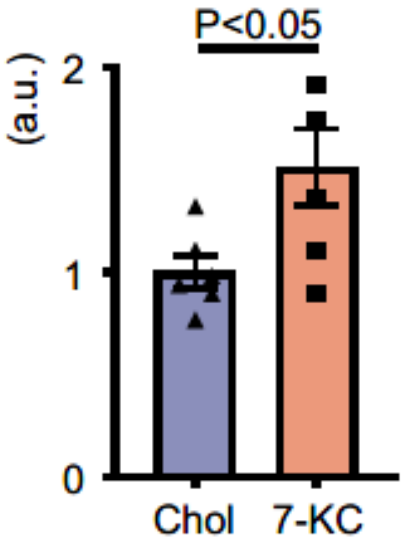

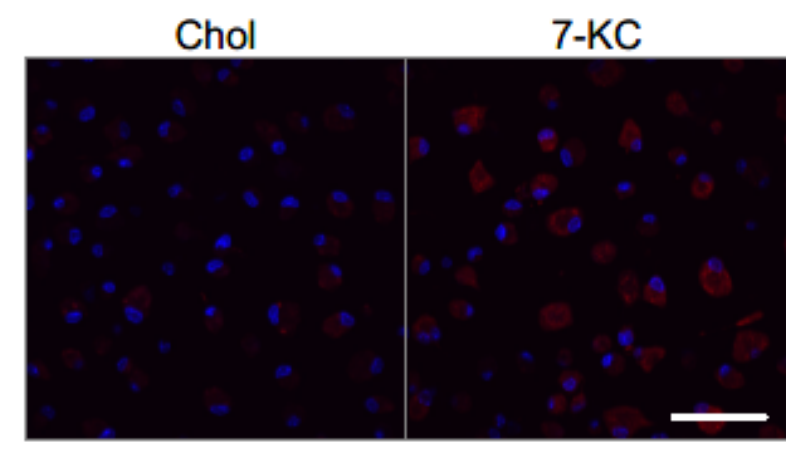

MitosoX

(Red)

DAPI

(Blue)

\section{Mitochondrial ROS}

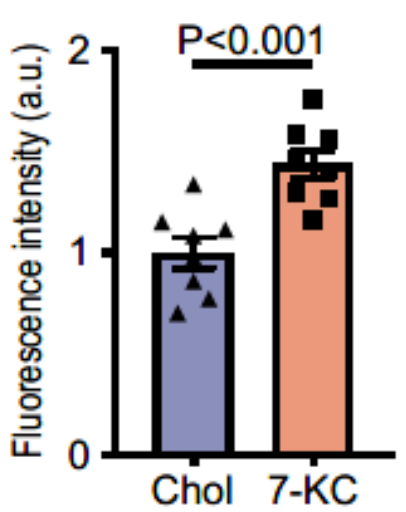

E

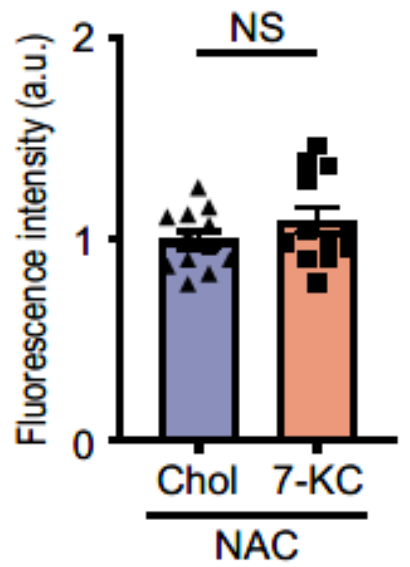

MCP-1 mRNA

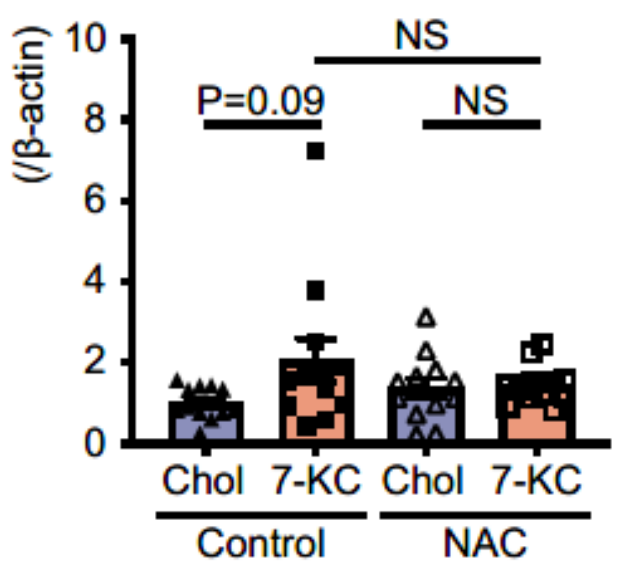

TNF- $\alpha$ mRNA

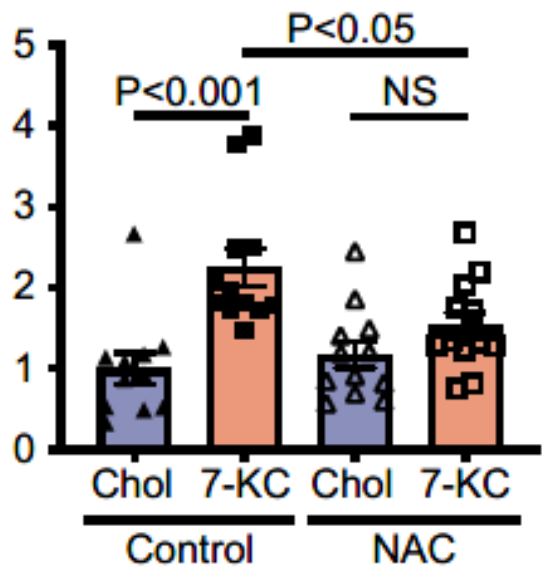

Figure 4 
7-KC induced inflammation and ROS production. A-C, Peritoneal macrophages were treated with vehicle, $10 \mu \mathrm{M}$ cholesterol or 7-KC for 24 hours. (A) Expression of cytokines quantified by RT-PCR. N=8. (B) p65 in the nuclear fraction quantified by ELISA to represent NF-KB activity. $N=6$. (C) Mitochondrial ROS measured using MitoSOX. $\mathrm{N}=8$. Scale bar, $50 \mu \mathrm{m}$. $\mathrm{D}$ and $\mathrm{E}$, Peritoneal macrophages were pretreated with $5 \mathrm{mM}$ NAC for 1 hour before $10 \mu \mathrm{M}$ cholesterol or 7-KC treatment for 24 hours. (D) Mitochondrial ROS measured using MitoSOX. $N=12$. (E) Expression of cytokines quantified by RT-PCR. $N=11-12$. Veh, vehicle; Chol, cholesterol; 7-KC, 7-ketocholesterol; ROS, reactive oxygen species; NAC, N-acetyl-L-cysteine. 
A

\begin{tabular}{c|l}
\multicolumn{1}{c|}{ Chol } \\
PERK & \\
BiP & \\
CHOP & \\
$\beta$-actin &
\end{tabular}
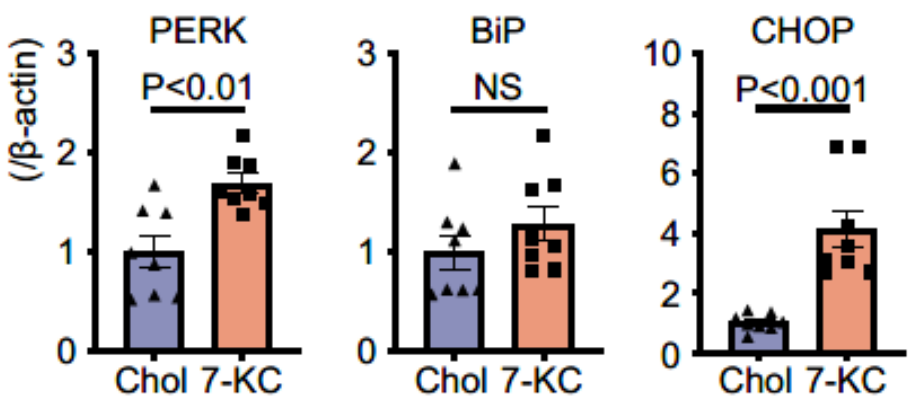

B

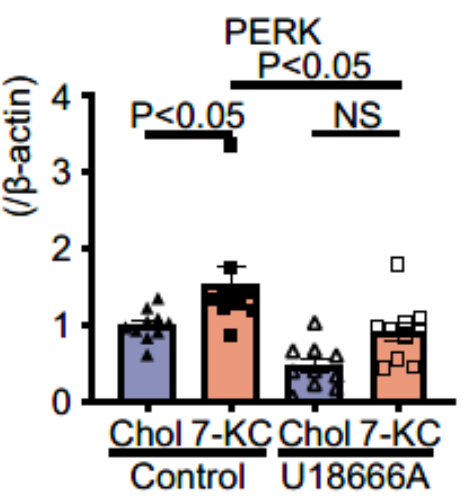

C Mitochondrial ROS

D
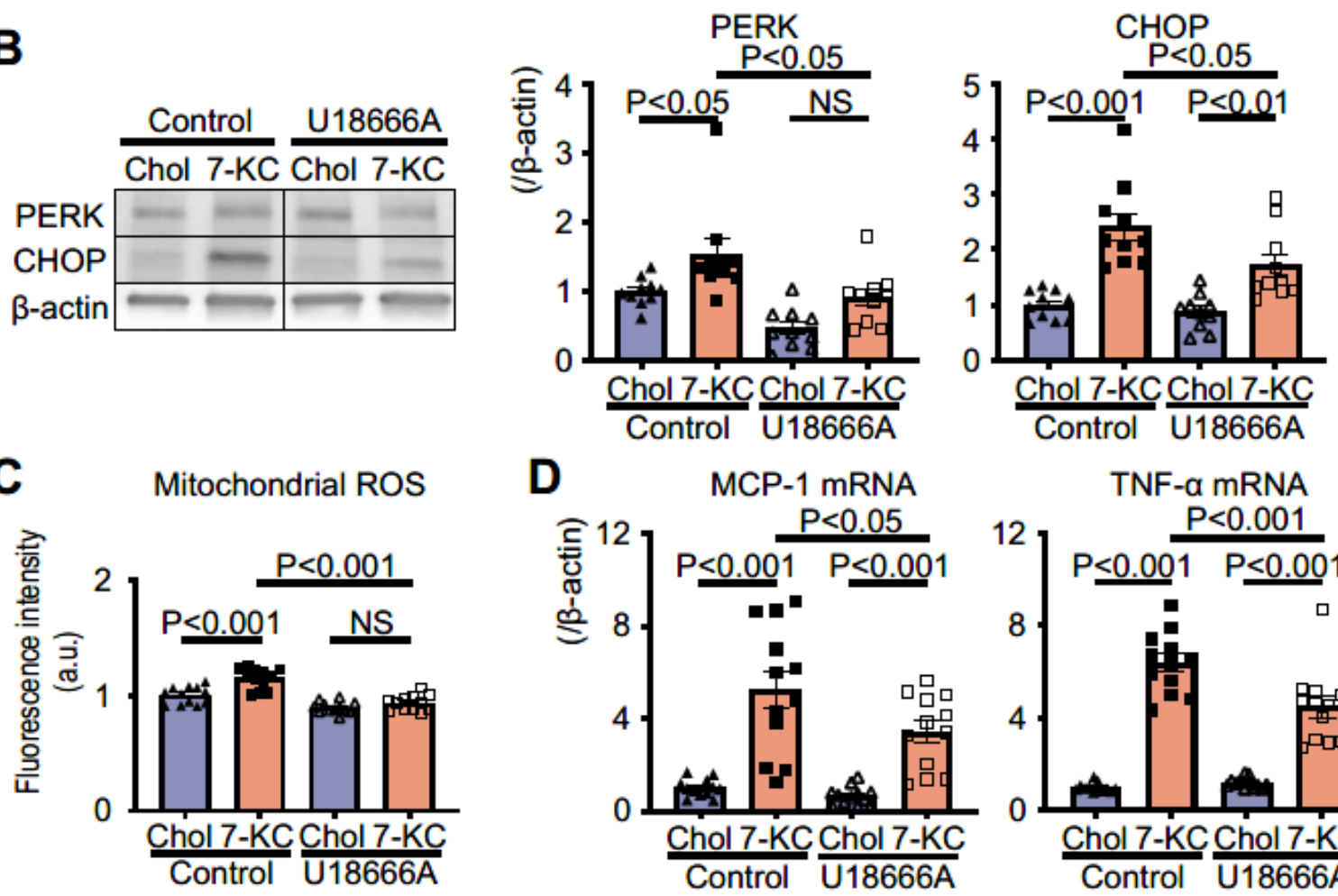

TNF- $\alpha$ mRNA

$\mathbf{E}$
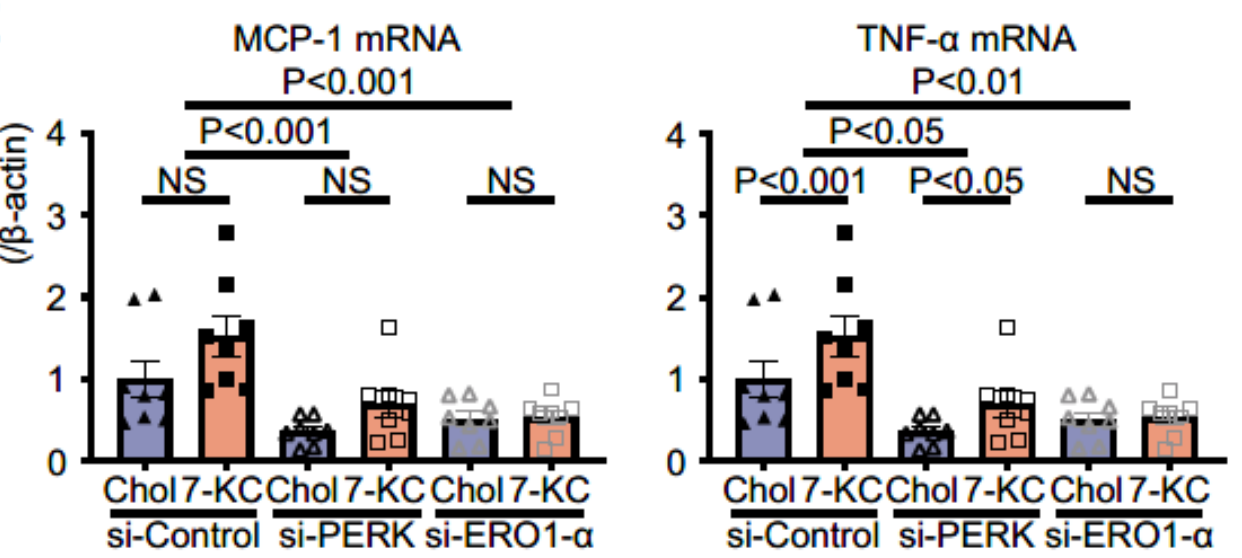

Figure 5

7-KC induced ER stress and subsequently ROS. A, Proteins related to ER stress in peritoneal macrophages treated with $10 \mu \mathrm{M}$ cholesterol or $7-\mathrm{KC}$ for 24 hours. $\mathrm{N}=8$. Original blots/gels are presented in Supplementary Figure S7. B-D, Effect of the cholesterol transport inhibitor U18666A on peritoneal macrophages pretreated with $3 \mu \mathrm{M}$ U18666A for 1 hour. (B) Proteins related to ER stress. $\mathrm{N}=10$. Original blots/gels are presented in Supplementary Figure S8. (C) Mitochondrial ROS measured using MitoSOX. 
$\mathrm{N}=12$. (D) Expression of cytokines quantified by RT-PCR. $\mathrm{N}=12$. E, Effect of siRNA specific for PERK or ER01-a. Expression of cytokines quantified by RT-PCR. N=8. P values were obtained by two-way ANOVA. Chol, cholesterol; 7-KC, 7-ketocholesterol; PERK, PKR-like endoplasmic reticulum kinase; BiP, binding immunoglobulin protein; $\mathrm{CHOP}, \mathrm{C} / \mathrm{EBP}$ homologous protein; ROS, reactive oxygen species; ER, endoplasmic reticulum; ERO1-a, ER oxidase 1 a; si-Control, nontargeted control siRNA; si-PERK, siRNA against PERK; si-ERO1-a, siRNA against ERO1-a.

\section{Supplementary Files}

This is a list of supplementary files associated with this preprint. Click to download.

- SupplementaryMaterialSciRep.pdf 